\title{
Leiomyoma with KAT6B-KANSL1 fusion: case report of a rapidly enlarging uterine mass in a postmenopausal woman
}

\author{
Alessandra J. Ainsworth ${ }^{1 *}$ (D), Nooshin K. Dashti ${ }^{2}$, Taofic Mounajjed ${ }^{2}$, Karen J. Fritchie ${ }^{2}$, Jaime Davila ${ }^{2}$, \\ Rohini Mopuri ${ }^{2}$, Rory A. Jackson ${ }^{2}$, Kevin C. Halling ${ }^{2}$, Jamie N. Bakkum-Gamez ${ }^{1}$ and J. Kenneth Schoolmeester ${ }^{2}$
}

\begin{abstract}
Background: Uterine leiomyomas, in contrast to sarcomas, tend to cease growth following menopause. In the setting of a rapidly enlarging uterine mass in a postmenopausal patient, clinical distinction of uterine leiomyoma from sarcoma is difficult and requires pathologic examination.

Case presentation: A 74-year-old woman presented with postmenopausal bleeding and acute blood loss requiring transfusion. She was found to have a rapidly enlarging uterine mass clinically suspicious for sarcoma. An abdominal hysterectomy and bilateral salpingo-oophorectomy were performed. A $15.5 \mathrm{~cm}$ partially necrotic intramural mass was identified in the uterine corpus. The tumor was classified as a cellular leiomyoma. RNA sequencing identified a KAT6B-KANSL1 fusion that was confirmed by RT-PCR and Sanger sequencing. After 6 months of follow-up, the patient remains asymptomatic without evidence of disease.

Conclusion: Prior studies of uterine leiomyomas have identified KAT6B (previously MORF) rearrangements in uterine leiomyomas, but this case is the first to identify a KAT6B-KANSL1 gene fusion in a uterine leiomyoma. While alterations of MED12 and HMGA2 are most common in uterine leiomyomas, a range of other genetic pathways have been described. Our case contributes to the evolving molecular landscape of uterine leiomyomas.
\end{abstract}

Keywords: KAT6B-KANSL1, Leiomyoma, Uterus

\section{Background}

Uterine leiomyomas are benign smooth muscle tumors found in nearly $70 \%$ of women by age 50 . These tumors can cause significant symptoms related to heavy menstrual bleeding and pelvic pressure, leading many women to pursue hysterectomy. After menopause, almost all women will have a reduction in both size and number of leiomyomas [1]. Conversely, uterine sarcomas classically present as a rapidly enlarging pelvic mass with or without vaginal bleeding or pelvic pain and are more commonly found in postmenopausal women [2]. The clinical distinction of uterine leiomyoma from sarcoma is difficult and definitive diagnosis is possible only by

\footnotetext{
* Correspondence: ainsworth.alessandra@mayo.edu

${ }^{1}$ Department of Obstetrics and Gynecology, Mayo Clinic, 200 First Street SW, Rochester, MN 55905, USA

Full list of author information is available at the end of the article
}

pathologic examination. We present an unusual case of a rapidly enlarging uterine mass in a postmenopausal patient that, following hysterectomy, was classified as a cellular leiomyoma. Subsequent molecular genetic and cytogenetic analysis of the tumor identified a $K A T 6 B-$ KANSL1 gene fusion. The only prior reporting of this fusion transcript was a retroperitoneal leiomyoma that morphologically and genetically resembled a uterine leiomyoma [3].

\section{Case presentation}

A 74 year-old Caucasian multiparous female with a history of ductal carcinoma in situ (DCIS) of the breast presented with significant postmenopausal bleeding, requiring blood transfusion, and a rapidly enlarging pelvic mass. DCIS was diagnosed 6 months prior to presentation and treated by unilateral total mastectomy and 
Tamoxifen which was transitioned to anastrozole at the onset of postmenopausal bleeding. She had a known history of uterine leiomyomas and no family history of uterine malignancy. Abdominal imaging by ultrasound and computerized tomography $(\mathrm{CT})$ revealed a $15 \mathrm{~cm}$ heterogeneous mass located centrally within the anterior mid body of the uterus (Fig. 1). The patient underwent total abdominal hysterectomy and bilateral salpingo-oophorectomy.

The uterus with attached bilateral fallopian tubes and ovaries weighed $635 \mathrm{~g}$. Grossly, the uterus contained a $15.5 \mathrm{~cm}$ well-demarcated intramural mass. The cut surface was white-tan to yellow with regions of necrosis. One section per centimeter of tumor was evaluated. Microscopically, the tumor was a cellular spindle cell neoplasm with anastomosing fascicles interrupted by thick-walled blood vessels or fibrous regions (Fig. 2a). A wispy or delicate hyaline extracellular matrix was seen throughout the tumor (Fig. 2b). The tumor cells had moderate eosinophilic cytoplasm, round to ovoid nuclei with fine chromatin and small nucleoli. The cells exhibited uniformly mild cytologic atypia. The mitotic index was no greater than 2 figures per 10 high power fields. Ischemic/hyaline-type necrosis was present, but no

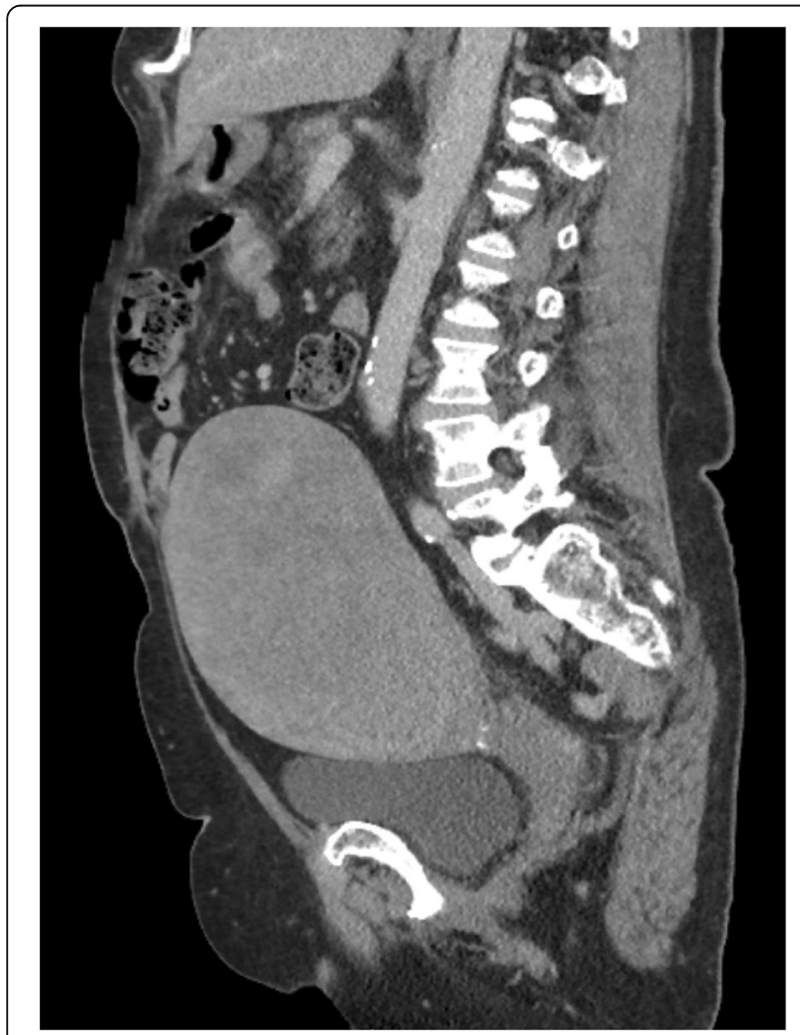

Fig. $1 \mathrm{CT}$ pelvis showing the large necrotic uterine mass evidence of tumor cell/coagulative necrosis was identified. Immunohistochemically, the tumor strongly and diffusely expressed desmin and h-caldesmon and exhibited patchy, strong expression of CD10. Aside from two benign endometrial polyps, the remainder of the specimen was unremarkable. The intramural mass was classified as a cellular leiomyoma. The patient's post-operative course was uneventful, and six months after surgery she remains asymptomatic without recurrence. She elected to discontinue all hormonal modulator therapy.

At the time of grossing, a sample of tumor was submitted for chromosomal karyotyping and RNA sequencing (RNA-Seq) according to a previously described protocol [4]. In brief, mRNA isolation, cDNA synthesis and library preparation utilized Illumina TruSeq Library Preparation Kit version 2 according to the manufacturer's protocol. Sequencing was performed by the Illumina HiSeq 2500. A customized bioinformatics pipeline for RNA-Seq analysis known as MAP-RSeq was used to assess fusions and gene expression [5]. RNA-Seq gene expression analysis compared gene expression by the tumor to normal uterine tissue from the Genotype-Tissue Expression (GTEX) database (https://www.gtexportal.org/home/).

A conventional karyotype showed complex chromosomal abnormalities, which included numerous structural and numeric abnormalities: 45-46, X,der(3)t(1;3)(q21;q26), $\mathrm{t}(5 ; 15)(\mathrm{q} 31 ; \mathrm{q} 22)$ add (6)(q25), ins(10;?)(q22;?),ins (11)(p15q21q23),+0-1mar[cp20]. RNA-Seq revealed a gene fusion involving KAT6B (10q22.2) and KANSL1 (17q21.31). The fusion joined exon 3 of $K A T 6 B$ to exon 11 of KANSL1. RT-PCR generated the expected fragment size of $165 \mathrm{bp}$ and Sanger sequencing confirmed the fusion (Fig. 3). Gene expression analysis of MED12, HMGA1 and HMGA2 revealed overexpression of HMGA2 and HMGA1 and normal expression of MED12 relative to normal uterine tissue (Fig. 4).

\section{Discussion}

Alterations of mediator complex subunit 12 (MED12) and high mobility group AT-hook 2 (HMGA2) are the most common genetic aberrations in uterine leiomyomas [6, 7]. MED12 is part of a multiprotein complex that acts to phosphorylate the C-terminal of RNA polymerase II. Missense mutations and in-frame insertion-deletions of $M E D 12$, frequently involving exon 2, disrupt the interaction of this complex and lead to diminished activity of a cyclin-dependent kinase and therefore decreased inhibition of transcription [6]. Aberrant function of MED12 has been shown to account for $70 \%$ of uterine leiomyomas [6, 7]. HMGA2 is an oncogene and functions through a cell 

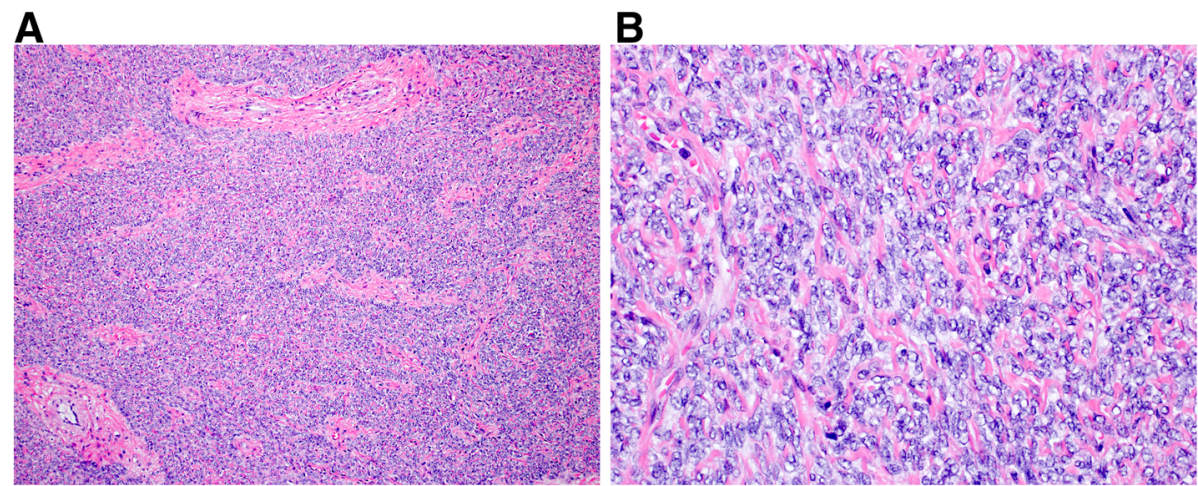

Fig. 2 Hematoxylin and eosin photomicrographs of the large uterine cellular leiomyoma. The tumor formed anastomosing fascicles with intermixed thick-walled blood vessels and small fibrous zones (a, 100x). The cells had uniformly mild cytologic atypia with eosinophilic cytoplasm, round to ovoid nuclei with fine chromatin and small nucleoli (b, 400x)

cycle checkpoint in the G1/S phase [8]. Tumors with HMGA2 translocations represent larger, solitary lesions compared to those with $M E D 12$ mutations [9].

Until recently, MED12 and $H M G A 2$ were thought to be mutually exclusive genetic events since no uterine leiomyomas had been found to harbor both alterations. HMGA2 translocations were described in $45 \%$ of tumors not containing MED12 mutations [10], suggesting multiple, less prevalent, genetic aberrations are responsible for the remaining 55\% of non-MED12 altered leiomyomas. However, a study by Galindo et al. found multiple leiomyomas containing both MED12 and HMGA2 alterations also had the greatest degree of complex chromosomal rearrangements [11]. The authors proposed that HMGA2 overexpression may be a consequence of these rearrangements $[10,11]$.
Monocytic leukemia zinc finger protein-related factor $(M O R F)$ located at $10 \mathrm{q} 22$, now known as KAT6B, is involved in histone acetyltransferase and allows segments of DNA to be more accessible to transcription factors. Moore and colleagues [12] previously described rearrangement of MORF in four uterine leiomyomas. In three tumors, they also mapped a break involving $17 \mathrm{q} 21$ but did not identify a gene fusion partner. The fusion of KAT6B and KANSL1 (the latter positioned at $17 \mathrm{q} 21$ ) has been identified only once previously in a retroperitoneal leiomyoma [3]. It has been proposed that KAT6B-KANSL1 might function in regulation of transcription [3]. Although $K A T 6 B$ rearrangements have been reported in leiomyomas, rare examples of uterine leiomyosarcoma [13] and acute myeloid leukemia [3, 12], fusions involving KANSL1 are rare. However, the three

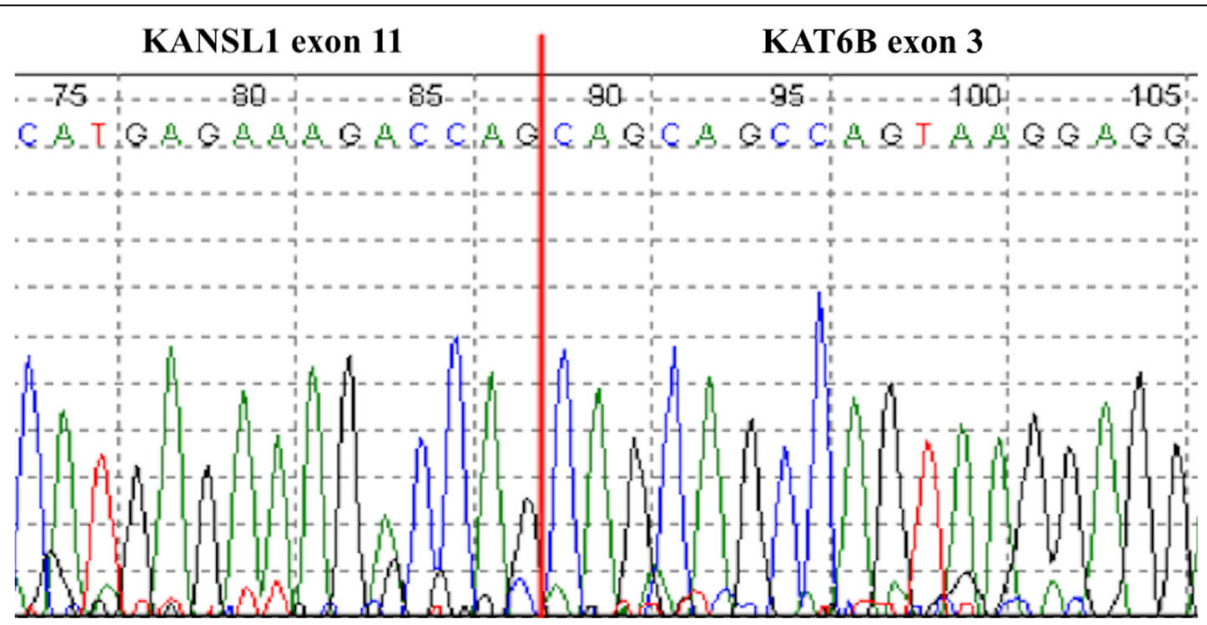

Fig. 3 Sanger sequencing confirmation (reverse direction) of KAT6B-KANSL1 sequence consistent with fusion of exon 3 of KAT6B to exon 11 of KANSL 1 


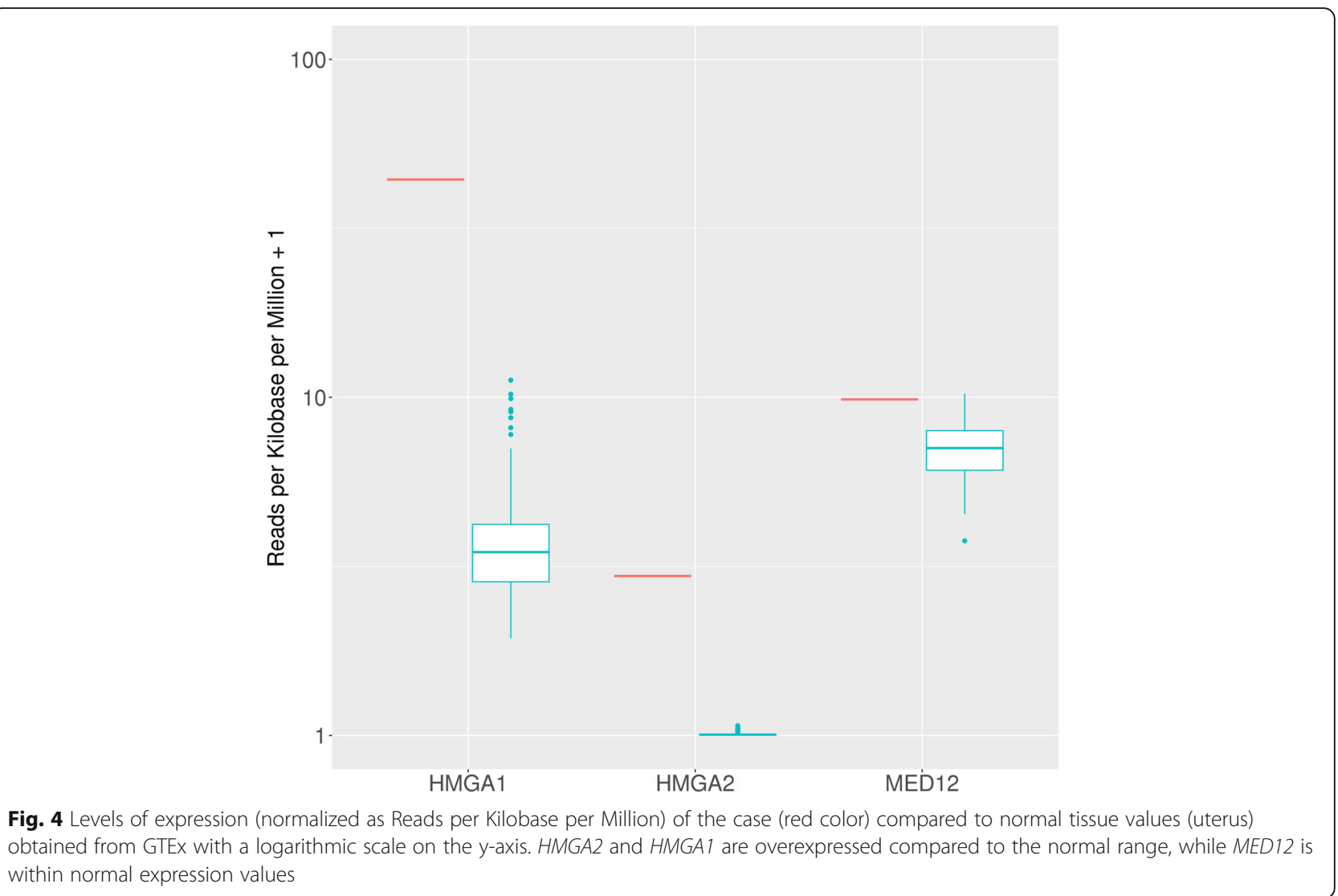

leiomyomas described by Moore et al. with a $17 q 21$ rearrangement likely are tumors with KAT6B-KANSL1 fusion [12]. A study of 9 uterine leiomyomas by Ozisik and investigators also identified 10q22 as a site of translocation in 8 typical leiomyomas and 1 cellular leiomyoma [14].

Recent advances in the genetics of leiomyoma tumorigenesis have proposed various pathways by which to categorize these tumors. A recent review organized genotypic and phenotypic alterations into categories of constitutional variants, somatic alterations and epigenetic mechanisms [15]. Constitutional variants refer to hereditary tumor susceptibility syndromes, including hereditary leiomyomatosis and renal cell carcinoma (HLRCC) and Alport Syndrome. Somatic alterations allow for classification by molecular subtype, broadly stratifying into structural abnormalities and mutations. Lastly, epigenetic mechanisms describe gene expression influences such as methylation. Another study that characterized the genetic events underlying uterine leiomyomas by means of whole genome sequencing and gene expression analysis uncovered four distinctive pathways, ranging from oncogenic stress from $M E D 12$ aberrations, metabolic stress from inactivation of $F H$ to complex chromosomal rearrangements induced by chromothripsis-like events and a variety of simple gene rearrangements [16].

Our case is the first to identify a KAT6B-KANSL1 gene fusion in a uterine leiomyoma, a finding that contributes to the evolving molecular landscape of leiomyomas that may allow for tailored therapeutic intervention or development of targeted therapy.

\section{Abbreviations}

DCIS: Ductal carcinoma in situ; CT: Computerized tomography; MORF: Monocytic leukemia zinc finger protein-related factor;

MED12: mediator complex subunit 12; HMGA2: high mobility group AT-hook 2

\section{Acknowledgements}

Not applicable.

\section{Funding}

Not applicable.

\section{Availability of data and materials}

Not applicable.

\section{Authors' contributions}

Each author significantly contributed to the critical aspects of this manuscript, and consequently, preparation of this manuscript would not have been possible without the contributions of each author listed. Each author edited and approved the final submitted version of the manuscript.

Ethics approval and consent to participate

No ethics approval was required for presentation of this case. 


\section{Consent for publication}

The patient agreed to publication of her case.

\section{Competing interests}

The authors declare that they have no competing interests.

\section{Publisher's Note}

Springer Nature remains neutral with regard to jurisdictional claims in published maps and institutional affiliations.

\section{Author details}

'Department of Obstetrics and Gynecology, Mayo Clinic, 200 First Street SW, Rochester, MN 55905, USA. ${ }^{2}$ Department of Laboratory Medicine and Pathology, Mayo Clinic, Rochester, MN, USA.

Received: 8 January 2019 Accepted: 10 April 2019

Published online: 25 April 2019

\section{References}

1. Wise LA, Laughlin-Tommaso SK. Epidemiology of uterine fibroids: from menarche to menopause. Clin Obstet Gynecol. 2016;59(1):2-24.

2. Santos P, Cunha TM. Uterine sarcomas: clinical presentation and MR features. Diagn Interv Radiol (Ankara, Turkey). 2015;21(1):4-9.

3. Panagopoulos I, Gorunova L, Bjerkehagen B, Heim S. Novel KAT6B-KANSL fusion gene identified by RNA sequencing in retroperitoneal leiomyoma with $\mathrm{t}(10 ; 17)(q 22 ; q 21)$. PLoS One. 2015:10(1):e0117010.

4. Davila Jl, Fadra NM, Wang X, McDonald AM, Nair AA, Crusan BR, et al. Impact of RNA degradation on fusion detection by RNA-seq. BMC Genomics. 2016;17(1):814.

5. Kalari KR, Nair AA, Bhavsar JD, O'Brien DR, Davila JI, Bockol MA, et al. MAP-RSeq: Mayo analysis pipeline for RNA sequencing. BMC Bioinformatics. 2014;15:224.

6. Mehine M, Makinen N, Heinonen HR, Aaltonen LA, Vahteristo P. Genomics of uterine leiomyomas: insights from high-throughput sequencing. Fertil Steril. 2014;102(3):621-9.

7. Makinen N, Mehine M, Tolvanen J, Kaasinen E, Li Y, Lehtonen HJ, et al. MED12, the mediator complex subunit 12 gene, is mutated at high frequency in uterine leiomyomas. Science (New York, NY). 2011; 334(6053):252-5.

8. Hodge JC, Kim TM, Dreyfuss JM, Somasundaram P, Christacos NC, Rousselle $M$, et al. Expression profiling of uterine leiomyomata cytogenetic subgroups reveals distinct signatures in matched myometrium: transcriptional profilingof the $\mathrm{t}(12 ; 14)$ and evidence in support of predisposing genetic heterogeneity. Hum Mol Genet. 2012;21(10):2312-29.

9. Hennig Y, Deichert U, Bonk U, Thode B, Bartnitzke S, Bullerdiek J. Chromosomal translocations affecting 12q14-15 but not deletions of the long arm of chromosome 7 associated with a growth advantage of uterine smooth muscle cells. Mol Hum Reprod. 1999:5(12):1150-4.

10. Bertsch E, Qiang W, Zhang Q, Espona-Fiedler M, Druschitz S, Liu Y, et al. MED12 and HMGA2 mutations: two independent genetic events in uterine leiomyoma and leiomyosarcoma. Mod Pathol. 2014;27(8):1144-53.

11. Galindo $L$, Hernandez-Beeftink T, Salas A, Jung Y, Reyes R, de Oca FM, et al. HMGA2 and MED12 alterations frequently co-occur in uterine leiomyomas. Gynecol Oncol. 2018;150(3):562-8,

12. Moore SD, Herrick SR, Ince TA, Kleinman MS, Dal Cin P, Morton CC, et al. Uterine leiomyomata with $\mathrm{t}(10 ; 17)$ disrupt the histone acetyltransferase MORF. Cancer Res. 2004;64(16):5570-7.

13. Dal Cin P, Boghosian L, Crickard K, Sandberg AA. T(10;17) as the sole chromosome change in a uterine leiomyosarcoma. Cancer Genet Cytogenet. 1988;32(2):263-6.

14. Ozisik YY, Meloni AM, Surti U, Sandberg AA. Involvement of 10q22 in leiomyoma. Cancer Genet Cytogenet. 1993;69(2):132-5.

15. Ordulu Z. Fibroids: genotype and phenotype. Clin Obstet Gynecol. 2016 59(1):25-9.

16. Mehine M, Kaasinen E, Makinen N, Katainen R, Kampjarvi K, Pitkanen E, et al. Characterization of uterine leiomyomas by whole-genome sequencing. $\mathrm{N}$ Engl J Med. 2013;369(1):43-53.

Ready to submit your research? Choose BMC and benefit from:

- fast, convenient online submission

- thorough peer review by experienced researchers in your field

- rapid publication on acceptance

- support for research data, including large and complex data types

- gold Open Access which fosters wider collaboration and increased citations

- maximum visibility for your research: over $100 \mathrm{M}$ website views per year

At $\mathrm{BMC}$, research is always in progress.

Learn more biomedcentral.com/submissions 